\title{
High priests of precision
}

William H. Press

The Fundamental Physical Constants and the Frontier of Measurement.

By B.W. Petley.

Adam Hilger: 1984. Pp.346. £28.50, \$49.

IN $A$ by now time-worn story, an experimental physicist is asked by a pure mathematician to name a few prime numbers. The physicist replies that 3,5 and 7 are prime, 9 seems not to be, and 11 and 13 are prime - "hmm...nine must be experimental error". The fact that this is (was once?) funny reveals, I think, a couple of assumptions about science that we take for granted and rarely examine. First is the belief that mathematics is "exact", so that the very notion of experimental error is laughable. Second, more subtle, is the implicit assumption that physics can be made arbitrarily precise, revealing an underlying reality of fully mathematical exactitude: we little doubt that the experimental physicist in the story feels that he can build a better apparatus, eliminate the experimental error, and prove that 9 is a prime! To put it another way, I think that the joke loses some of its pith if "experimental psychologist" or "anthropologist" is substituted for "experimental physicist", and I mean this as no insult to my social-scientist colleagues, whose fields do not (and have no need to) pretend to the exactitude of physics.

In our time, only a small fraction of physicists work to uphold their discipline's reputation as an "exact" science. Search not for such individuals among the highenergy particle physicists at CERN, where important discoveries tend to be qualitative, as confirming the existence of the theoretically-predicted top quark or intermediate vector boson. Nor look, generally, to the solid-state physicists at Bell Labs; there too, the most interesting discoveries are qualitatively new material behaviours or transitions. For exact physics, look instead to Britain's National Physical Laboratory (where Dr Petley works), or, in the United States, to the National Bureau of Standards or affiliated Joint Institute for Laboratory Astrophysics, or to the laboratories of individual experimenters in small or large universities. These are the habitats of the "metrologist", whose intellectual world is the subject of Dr Petley's book.

Metrology is the physics of high-precision measurement. Is it truly fundamental science, or is it merely necessary, industrial standards-keeping? That question runs between the lines of this book. Not unexpectedly Dr Petley holds a brief on one side. Occasionally one finds his tone a bit too defensive, a bit too revealing of the fact that the metrologist, whether unfairly or not, ranks low in today's physics pecking order. The author's case against this prejudice is strongest not when he waxes intellectual about the matter, but rather when he describes the elegance and diversity of modern, high-precision experiments.

The question "fundamental or not" has some subtlety. A simple example is the speed of light. Special relativity, that essential framework on which all of modern physics rests, predicts and requires the speed of light $c$ to be absolutely units involving fundamental constants, for example, the Josephson effect for the volt and the quantised Hall conductance for the siemens, or ohm, such systems must be set up with care. If they are not, one finds that the [magnetic permeability of vacuum becomes] a constant which must be evaluated experimentally, as it did during the 1930s for a while". (Emphasis added.) For a while?! This is not ordinary science.

It is an arcane world, and Dr Petley is a fascinating guide, in spite of (or perhaps because of his tendency to dive enthusiastically into a welter of details which will leave non-physicist readers gasping for breath - but awestruck. If "God is in the details", then the high-precision metrologist is surely above the saints. Physicist readers will be able to follow the exposition, even when it is occasionally muddy, and will be repaid by learning a lot.

The book is organized into a couple of

\section{IMAGE \\ UNAVAILABLE FOR COPYRIGHT REASONS}

Record speed - the microwave interferometer used by K.D. Froome to measure the velocity of light at the National Physical Laboratory in 1957. His result formed the basis for the value recommended by the International Astronomical Union until it was superseded by the measurements which culminated in the redefinition of the metre in 1983.

constant, independent of where and how it is measured. In this light, high-precision measurement of $c$ seems fundamental indeed. Speed being a length per time, one must "bring" the standard second and standard metre to any laboratory in which $c$ is to be measured. The second (that is, the first) is relatively transportable, exemplified in caesium-133 atomic clocks and hydrogen masers. The metre has historically proved more difficult to transport or, for that matter, define. Its definition as a platinum-iridium bar in Paris gave way in 1960 to a certain number of wavelengths of krypton-86 light. During the 1970 s the overriding limitation on the measurement of $c$, whose value was now known to be something like $299,792,458.1 \pm 1.9 \mathrm{~m} \mathrm{~s}^{-1}$, became the accuracy with which a laboratory baseline length could be related to the krypton-86 standard. In 1983 the speed of light became defined as $299,792,458 \mathrm{~m} \mathrm{~s}^{-1}$; the metre lost its independent definition and is now derived from the standard of time. But consider the plight of the experimenter who was thought to be measuring $c$, which seemed fundamental, but is now revealed as having merely measured the standard metre, which sounds terribly industrial!

In this book one is plunged back and forth between the worlds of physics and international commerce: "Although it is tempting to set up a system of electrical introductory and philosophical chapters, followed by individual chapters on the "mechanical" and the "electrical" fundamental constants. There is a chapter on high-precision tests of quantum electrodynamics (most notably the Lamb shift), one on matters relating to gravitation, and one on so-called "null experiments" which test experimentally such tacit (and often over-looked) assumptions as that a body's inertia is the same no matter what the direction in which it is accelerated. Also included is discussion of some trendy topics, such as the large-number coincidences in cosmology and the prospects for detecting gravitational waves. But in truth these are superfluous, since the author is at his best when guiding us through real-world laboratory details: "Consequently, by setting on the side of this peak it was possible to check that the displacement of the crystal was in a straight line to $\pm 0.00006^{\prime \prime}$. In fact, there was a small curvature of $0.0017^{\prime \prime}$ which was taken into account ...". Read on, and you will learn how the "Siegbahn constant" is measured! It gives a precise calibration of the lattice spacing in silicon, needed in turn for accurate determination of the Avogadro constant so fundamental to physical chemistry.

William H. Press is Professor of Astronomy and of Physics at Harvard University. 